Case Report

\title{
Therapeutic Management of a Substantial Pelvic Aneurysmatic Bone Cyst Including the Off-Label Use of Denosumab in a 35-Year-Old Female Patient
}

\author{
D. Ntalos, ${ }^{1}$ M. Priemel, ${ }^{1}$ C. Schlickewei, ${ }^{1}$ D. M. Thiesen, ${ }^{1}$ J. M. Rueger, ${ }^{1}$ and A. S. Spiro ${ }^{2}$ \\ ${ }^{1}$ Department of Trauma, Hand, and Reconstructive Surgery, University Medical Center Hamburg-Eppendorf, Hamburg, Germany \\ ${ }^{2}$ Department of Pediatric Orthopedic Surgery, Children's Hospital Hamburg-Altona, Hamburg, Germany
}

Correspondence should be addressed to D. Ntalos; d.ntalos@uke.de

Received 3 August 2017; Accepted 19 September 2017; Published 17 October 2017

Academic Editor: Akio Sakamoto

Copyright (c) 2017 D. Ntalos et al. This is an open access article distributed under the Creative Commons Attribution License, which permits unrestricted use, distribution, and reproduction in any medium, provided the original work is properly cited.

\begin{abstract}
Aneurysmal bone cysts $(\mathrm{ABC})$ are benign bone tumors, which are highly vascularized. The main course of treatment is curettage followed by bone grafting or cement insertion. Still recurrence remains a main problem for patients. Denosumab is a monoclonal antibody, which acts as an inhibitor of the RANK/RANKL pathway, diminishing bone turnover. Recent case reports have shown that Denosumab can be a promising therapeutic agent for people suffering from therapy-resistant ABC. We report the case of a 35-year-old female patient presenting with a pronounced ABC of the pelvis. Since the tumor was inoperable, Denosumab was administered, leading to a significant shrinkage of the lesion, which allowed surgical intervention. Upon recurrence, Denosumab was restarted putting the patient once more into remission. Follow-up was four years overall with a clinical and radiological stable disease for fifteen months after final discontinuation of the monoclonal antibody. Therefore, our case further underlines the potential of Denosumab in the treatment of $\mathrm{ABC}$.
\end{abstract}

\section{Introduction}

Aneurysmal bone cysts (ABC) were first described in 1942 by Jaffe and Lichtenstein and account for almost one per cent of all benign bone tumors $[1,2]$. Typically, the tumor occurs in the first two decades of a person's life, though up to twenty per cent are diagnosed later on [3]. ABC are highly vascular, eccentric, osteolytic lesions of unknown origin which most commonly manifest themselves in the metaphysis of the long bones, especially the femur and tibia $[4,5]$. Involvement of the pelvis is rather rare and occurs, depending on the study, in four to twelve per cent of the cases $[2,4,5]$. The pathogenesis of these lesions, although having been known now for more than seventy years, still remains unclear. Among vascular and traumatic factors, recent studies describe genetic alterations as well $[3,6,7]$. Treatment options for aneurysmal bone cysts comprise surgical resection or curettage followed by bone grafting or cement insertion as well as arterial embolization or direct injection of the cyst with sclerosing agents such as polidocanol or local injection of doxycycline [8-10]. Focusing on the pelvis, optimal treatment is challenging since the lesions are usually very large, highly vascularized and the anatomical relation to the acetabulum and neurovascular structures reduces the chance of adequate resection $[2,11,12]$.

As a possible new therapeutic agent for $\mathrm{ABC}$, the use of Denosumab has been described to our knowledge in only five cases so far. Denosumab operates as an inhibitor of RANKL, which is known to increase bone turnover due to stimulating osteoclastic cells. The monoclonal antibody has therefore been implemented in the treatment of osteoporosis, skeletal metastases, and giant cell tumors of the bone with good results. In context of aneurysmal bone cysts, there is still a lack of data. The five existing cases show very promising results for Denosumab being a treatment option for $A B C$ though, which is in accordance with our findings [12-16].

\section{Case Report}

A 35-year-old female patient presented herself as an outpatient in 2012 with a two-week history of pain in the right leg 




FIGURE 1: Initial MR and CT Imaging showing a substantial right-sided cystic tumor of the pelvis infiltrating associated neuroforamina.

and hyposensibility of the right calf. For further investigation, an MRI of the lumbar spine was performed showing a rightsided cystic tumor of the pelvis instead of a suspected disc herniation. In the following, an abdominal CT scan was performed revealing an osteolytic involvement of the right ileum, os sacrum, and the right-sided neuroforamina s1-s4 (Figure 1).

On physical examination, the patient showed a moderate swelling of the right gluteal region with a slight reduction in range of motion of the right hip due to severe pain during leg movements. Otherwise the exam was unremarkable with normal blood circulation in both legs and intact sensitivity except for the right dorsal calf.

To further evaluate the tumor, an MRI scan of the pelvis and an open biopsy were performed (Figure 1).

The suspected diagnosis of an aneurysmal bone cyst was confirmed by histopathological examination. Treatment options were discussed in an interdisciplinary tumor board. Surgical intervention was not recommended due to inaccessibility. Therefore, selective arterial embolization was performed by our radiologists. Additionally, the off-label use of $60 \mathrm{mg}$ of Denosumab, once every four weeks, was initiated. It was decided to start the patient with $60 \mathrm{mg}$ and not $120 \mathrm{mg}$ to assess a potential therapeutic effect while keeping sideeffects low. Due to the unstable situation of the right pelvis,

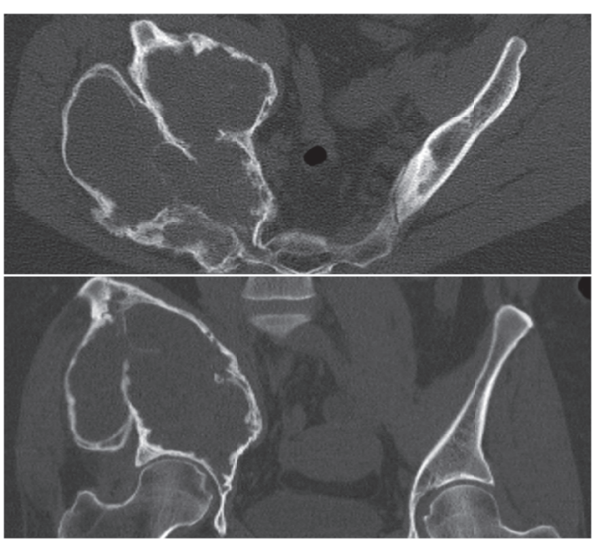

FIgURE 2: Preoperative follow-up CT scan showing a notable increase in cortical thickness.

the patient was advised to use crutches with no weight bearing of the right leg. Follow-up examination and CT imaging were performed every three months and revealed a significant reduction in pain and a notable increase in cortical thickness, while less mineralization of the cyst cavity was seen (Figure 2). Therefore, Denosumab dosages were not changed. Additionally, an increase to partial weight bearing of the leg 


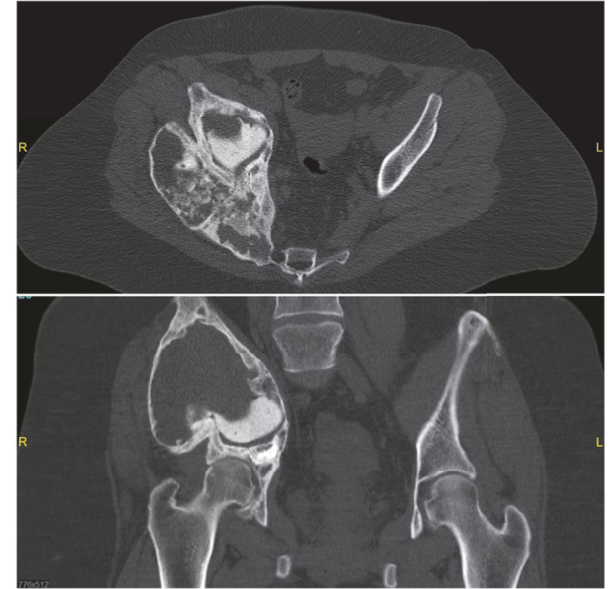

Figure 3: Follow-up CT scan of the pelvis showing stable disease after discontinuation of the second cycle of Denosumab treatment for fifteen months.

up to $20 \mathrm{~kg}$ was possible and therapy with Denosumab was discontinued after a total course of twelve months. During this time no remarkable side-effects were detected. During interdisciplinary discussions, surgical treatment via curettage and a combination of bone grafting and cement implantation of the defect was planned. Prior to surgery embolization of the remaining arteries was performed again.

Surgery included curettage, implantation of autologous spongiosa, alloplastic bone grafts, artificial bone material, and bone cement.

A follow-up CT scan six months after surgery revealed a significant tumor progress with decreased cortical thickness. Since there was a good response to Denosumab before, the treatment was restarted.

Follow-up imaging showed a significant response with tumor reduction, recalcification, and increasing stability of the pelvis again. Thus Denosumab therapy was continued, controlling the aforementioned lesion and allowing the patient to fully bear weight on the said leg. In the following, laboratory tests revealed signs of low turnover osteoporosis with low calcium, phosphate, and 25-hydroxyvitamin D3 levels combined with increased parathyroid hormone $(\mathrm{PTH})$ levels resulting in discontinuation of Denosumab after a total of 17 months during the second cycle. Until today the overall patient follow-up is about four years with clinical examinations being performed in our outpatient clinic every 6-12 months. Denosumab has been discontinued for fifteen months with clinical and radiological examination showing a stable disease so far (Figure 3).

\section{Discussion}

There are several treatment options for aneurysmal bone cysts. Surgical treatment includes en bloc resection or curettage combined with bone grafting or cement application $[2,11,12,17]$. Either one can be combined with neoadjuvant or adjuvant treatment. Additionally, nonsurgical management using cryotherapy, sclerotherapy, doxycycline injections, radionuclide ablation, arterial embolization, or radiotherapy has been described to yield good results as well. Despite these various options recurrence of $\mathrm{ABC}$ remains a main problem $[2,11,12,17]$.

Resecting the tumor with wide margins has been described to have the lowest rate of recurrence but due to anatomical reasons it is rarely possible, as can be seen in our case. In this context, the effect of Denosumab can be used to diminish tumor size and enhance operability of the lesion. However, increasing bone mineralization and development of septal bone separations caused by Denosumab can complicate surgical treatment.

In our case, the patient relapsed within six months after surgical curettage and graft insertion. Data ascertained by Mankin et al. show recurrence rates up to $30 \%$ after curettage alone and close to $25 \%$ following curettage succeeded by allograft or cement implantation. Based on different risk factors such as younger age, aggressiveness of the lesion, and type of treatment, rates can be even higher leading up to $59 \%$ $[18,19]$. Therefore, the course of disease as displayed by our patient is quite common and calls for potential long-term intervention.

Lange et al. and Pauli et al. have shown that recurrent ABC could be well controlled by Denosumab application $[12,14]$. Our case supports these findings and in particular the use of Denosumab in inoperable and recurrent $\mathrm{ABC}$ as a promising alternative. Since we used a multimodal approach including repeated embolization, the treatment results may have been caused in part by embolization and not completely by Denosumab.

The underlying mechanism of the monoclonal antibody in context of $A B C$ remains unclear. Denosumab acts as an inhibitor within the RANKL/RANK (receptor activator of NF- $\kappa$ B ligand/receptor activator of NF- $\kappa$ B) pathway. Since RANKL works as an essential differentiation factor for osteoclasts, it is a widely accepted theory that bone resorption is stimulated by this pathway and therefore could be decreased by the use of Denosumab [13,20,21]. Clinical data has already shown the successful therapeutic use of Denosumab in the context of osteoporosis, bone metastases, and recently giant cell tumor, which share immunohistochemical attributes with ABC. Furthermore, recent studies suggested RANKL to play an important part in $\mathrm{ABC}$ as well which could explain Denosumab's effects on ABC [15, 22-24].

Treating ABC with Denosumab is thus not only theoretically astute but has shown promising results in a very small group of patients, with five reported cases so far [12-16].

Furthermore Denosumab was suggested to potentially be used as first-line treatment as shown by the case of a fiveyear-old boy responding very well to the singular use of the antibody [15].

Upon considering Denosumab as a treatment option, one has to take notice of the side-effects. Even though Denosumab seems to be a well-tolerated drug, especially when facing long-term treatment several side-effects are described, with the most noteworthy seeming to be osteonecrosis of the jaw (ONJ) [25-27]. One study has shown an occurrence rate of $5 \%$ of $\mathrm{ONJ}$ in patient undergoing treatment [25]. These numbers though were derived in a population taking 
twice the amount that our patient received and ONJ was not observed in other trials where lower dosages of Denosumab were administered [26-28]. Furthermore Denosumab has been associated with severe hypocalcemia, atypical femur fractures, and rebound bone resorption phenomena [29]. Therefore, considering that there are alternative treatment options such as polidocanol [30] and doxycycline injection [8], which seem to be very promising when it comes to inoperable $\mathrm{ABC}$, the usage of Denosumab should be carefully thought through.

In conclusion, Denosumab seems to be a promising new treatment alternative in $\mathrm{ABC}$, especially in patients where surgical resection is not an option and in relapsing patients where potential physical disabilities can thus be evaded.

\section{Consent}

Informed consent including publication of the manuscript as well as photographs has been obtained from the patient.

\section{Conflicts of Interest}

The authors declare that there are no conflicts of interest regarding the publication of this article.

\section{References}

[1] H. J. Mankin, F. J. Hornicek, E. Ortiz-Cruz, J. Villafuerte, and M. C. Gebhardt, "Aneurysmal bone cyst: A review of 150 patients," Journal of Clinical Oncology, vol. 23, no. 27, pp. 6756-6762, 2005.

[2] J. Cottalorda, F. Chotel, R. Kohler et al., "Aneurysmal bone cysts of the pelvis in children: A multicenter study and literature review," Journal of Pediatric Orthopaedics, vol. 25, no. 4, pp. 471475, 2005.

[3] R. Radulescu, A. Badila, R. Manolescu, M. Sajin, and I. Japie, "Aneurysmal bone cyst-clinical and morphological aspects," Romanian Journal of Morphology and Embryology, vol. 55, no. 3, pp. 977-981, 2014.

[4] A. Agarwal, P. Goel, S. A. Khan, P. Kumar, and N. A. Qureshi, "Large aneurysmal bone cyst of iliac bone in a female child: A case report," Journal of Orthopaedic Surgery and Research, vol. 5, no. 1, article no. 24, 2010.

[5] E. N. Novais, A. K. Zimmerman, L. W. Lewallen, P. S. Rose, F. H. Sim, and A. L. McIntosh, "Functional outcomes and quality of life following surgical treatment of aneurysmal bone cysts of the pelvis in children," Journal of Children's Orthopaedics, vol. 8, no. 3, pp. 281-288, 2014.

[6] A. M. Oliveira and M. M. Chou, "USP6-induced neoplasms: the biologic spectrum of aneurysmal bone cyst and nodular fasciitis," Human Pathology, vol. 45, no. 1, pp. 1-11, 2014.

[7] A. W. Lau, L. M. Pringle, L. Quick et al., “TRE17/ubiquitinspecific protease 6 (USP6) oncogene translocated in aneurysmal bone cyst blocks osteoblastic maturation via an autocrine mechanism involving bone morphogenetic protein dysregulation," The Journal of Biological Chemistry, vol. 285, no. 47, pp. 37111-37120, 2010.

[8] W. E. Shiels II and J. L. Mayerson, "Percutaneous doxycycline treatment of aneurysmal bone cysts with low recurrence rate: A preliminary report tumor," Clinical Orthopaedics and Related Research, vol. 471, no. 8, pp. 2675-2683, 2013.
[9] O. Brosjö, P. Pechon, A. Hesla, P. Tsagozis, and H. Bauer, "Sclerotherapy with polidocanol for treatment of aneurysmal bone cysts," Acta Orthopaedica, vol. 84, no. 5, pp. 502-505, 2013.

[10] O. Brosjö and P. Tsagozis, “Treatment of an aggressive aneurysmal bone cyst with percutaneous injection of polidocanol: a case report," Journal of Medical Case Reports, vol. 8, p. 450, 2014.

[11] T. B. Rapp, J. P. Ward, and M. J. Alaia, "Aneurysmal bone cyst," Journal of the American Academy of OrthopaedicSurgeons, vol. 20, no. 4, pp. 233-241, 2012.

[12] T. Lange, C. Stehling, B. Fröhlich et al., "Denosumab: A potential new and innovative treatment option for aneurysmal bone cysts," European Spine Journal, vol. 22, no. 6, pp. 1417-1422, 2013.

[13] H. Yasuda, "RANKL, a necessary chance for clinical application to osteoporosis and cancer-related bone diseases," World Journal of Orthopedics, vol. 4, no. 4, pp. 207-217, 2013.

[14] C. Pauli, B. Fuchs, C. Pfirrmann, J. A. Bridge, S. Hofer, and B. Bode, "Response of an aggressive periosteal aneurysmal bone cyst (ABC) of the radius to denosumab therapy," World Journal of Surgical Oncology, vol. 12, no. 1, article no. 17, 2014.

[15] D. W. Pelle, J. W. Ringler, J. D. Peacock et al., "Targeting receptor-activator of nuclear kappaB ligand in aneurysmal bone cysts: Verification of target and therapeutic response," Translational Research, vol. 164, no. 2, pp. 139-148, 2014.

[16] A. Dubory, G. Missenard, J. Domont, and C. Court, "Interest of denosumab for the treatment of giant-cells tumors and aneurysmal bone cysts of the spine. About nine cases," The Spine Journal, vol. 41, no. 11, pp. E654-E660, 2016.

[17] Z. Cheng, X. Peng, and W. He, "Arterial embolization of primary sacral aneurysmal bone cyst," Chinese Medical Journal, vol. 127, no. 9, pp. 1785-1787, 2014.

[18] J. P. Dormans, B. G. Hanna, D. R. Johnston, and J. S. Khurana, "Surgical Treatment and Recurrence Rate of Aneurysmal Bone Cysts in Children," Clinical Orthopaedics and Related Research, no. 421, pp. 205-211, 2004.

[19] K. Başarir, A. Pişkin, B. Güçlü, Y. Yildiz, and Y. Sağlik, "Aneurysmal bone cyst recurrence in children: a review of 56 patients," Journal of Pediatric Orthopaedics, vol. 27, no. 8, pp. 938-943, 2007.

[20] G. D. Roodman, "Cell biology of the osteoclast," Experimental Hematology, vol. 27, no. 8, pp. 1229-1241, 1999.

[21] T. Mizoguchi, A. Muto, N. Udagawa et al., "Identification of cell cycle-arrested quiescent osteoclast precursors in vivo," The Journal of Cell Biology, vol. 184, no. 4, pp. 541-554, 2009.

[22] R. M. I. Taylor, T. G. Kashima, F. K. E. Hemingway, A. Dongre, H. J. Knowles, and N. A. Athanasou, "CD14- mononuclear stromal cells support (CD14+) monocyte-osteoclast differentiation in aneurysmal bone cyst," Laboratory Investigation, vol. 92, no. 4, pp. 600-605, 2012.

[23] T. Yamagishi, H. Kawashima, A. Ogose et al., "Receptor-activator of nuclear kappaB ligand expression as a new therapeutic target in primary bone tumors," PLoS ONE, vol. 11, no. 5, Article ID e0154680, 2016.

[24] K. Y. Won, R. K. Kalil, Y. W. Kim, and Y.-K. Park, "RANK signalling in bone lesions with osteoclast-like giant cells," Pathology, vol. 43, no. 4, pp. 318-321, 2011.

[25] M. R. Smith, F. Saad, R. Coleman et al., "Denosumab and bone-metastasis-free survival in men with castration-resistant prostate cancer: results of a phase 3, randomised, placebocontrolled trial," The Lancet, vol. 379, no. 9810, pp. 39-46, 2012.

[26] G. K. Ellis, H. G. Bone, R. Chlebowski et al., "Randomized trial of denosumab in patients receiving adjuvant aromatase 
inhibitors for nonmetastatic breast cancer," Journal of Clinical Oncology, vol. 26, no. 30, pp. 4875-4882, 2008.

[27] P. Narayanan, "Denosumab: A comprehensive review," South Asian Journal of Cancer, vol. 2, no. 4, p. 272, 2013.

[28] M. R. Smith, B. Egerdie, N. H. Toriz et al., "Denosumab in men receiving androgen-deprivation therapy for prostate cancer," The New England Journal of Medicine, vol. 361, no. 8, pp. 745755, 2009.

[29] M. Pazianas and B. Abrahamsen, "Osteoporosis treatment: bisphosphonates reign to continue for a few more years, at least?" Annals of the New York Academy of Sciences, vol. 1376, no. 1, pp. 5-13, 2016.

[30] F. Batisse, A. Schmitt, T. Vendeuvre, D. Herbreteau, and C. Bonnard, "Aneurysmal bone cyst: A 19-case series managed by percutaneous sclerotherapy," Orthopaedics \& Traumatology: Surgery \& Research, vol. 102, no. 2, pp. 213-216, 2016. 


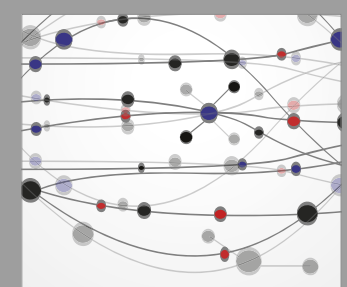

The Scientific World Journal
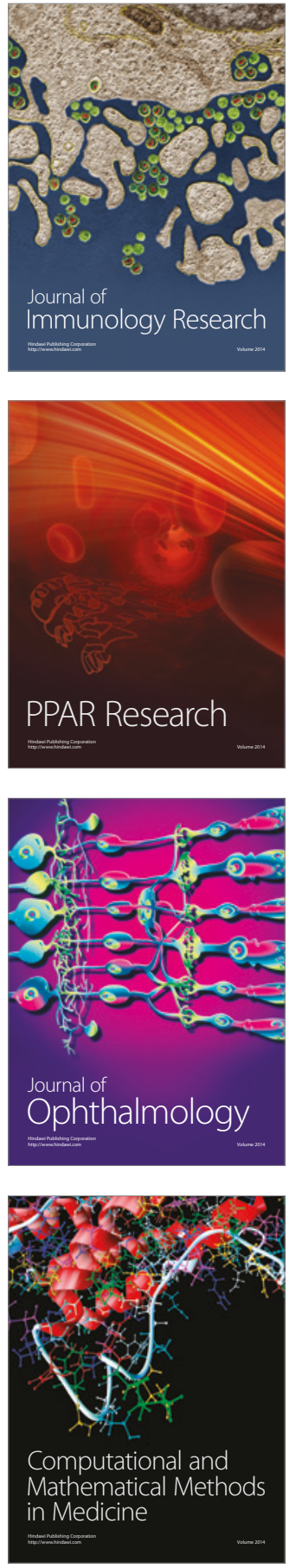

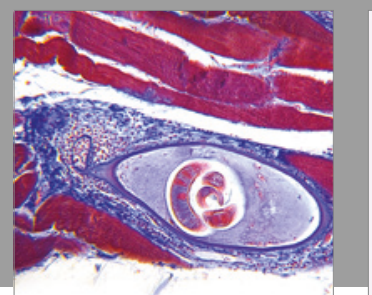

Gastroenterology Research and Practice
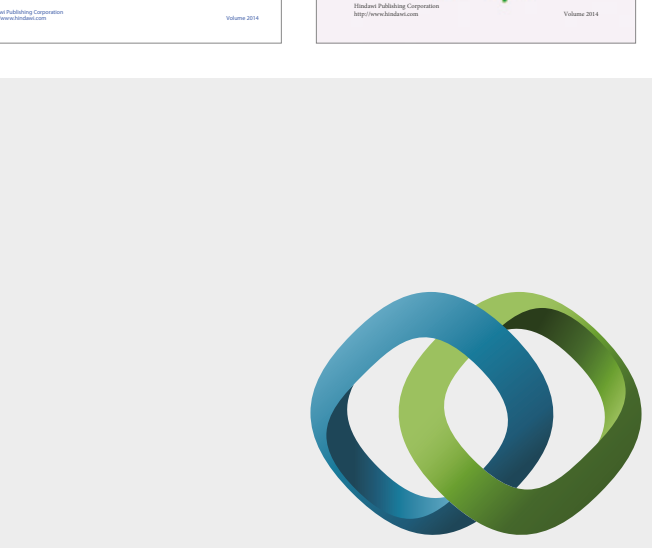

\section{Hindawi}

Submit your manuscripts at

https://www.hindawi.com
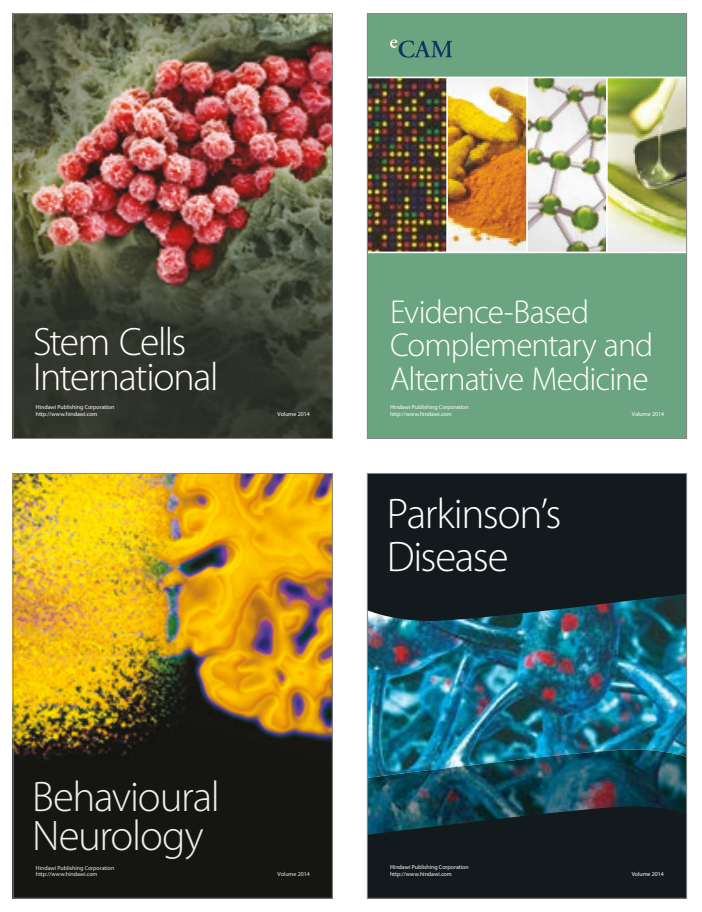
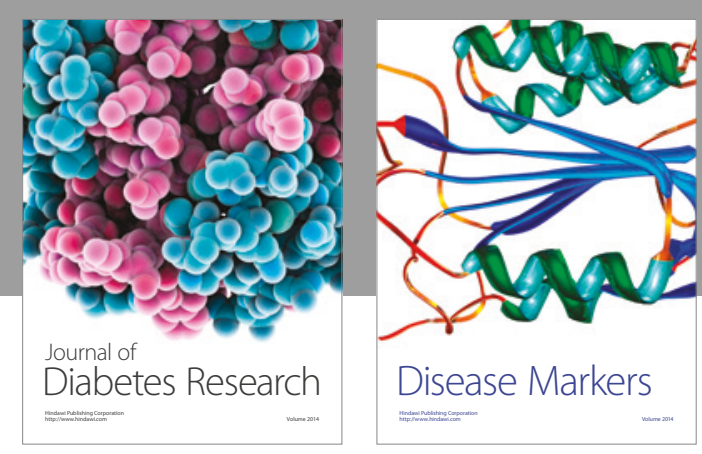

Disease Markers
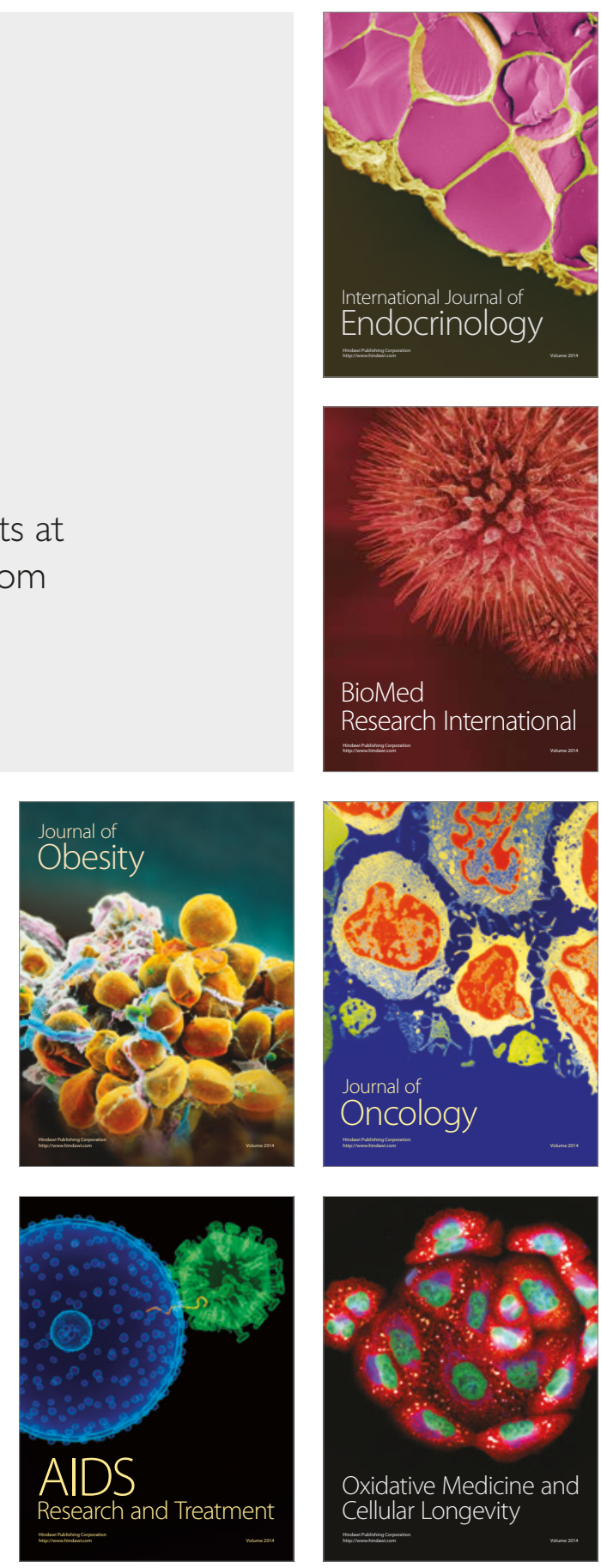